\title{
Properties of Fuel Spray Obtained by Electrohydrodynamic Atomization
}

\author{
Michel Daaboul*1, Nicolas Saba ${ }^{1}$, Jihad Rishmany ${ }^{1}$, Christophe Louste ${ }^{2}$ \\ ${ }^{1}$ Mechanical Engineering Department, University of Balamand, Lebanon \\ ${ }^{2}$ EHD Team, PPRIME Institute, CNRS - University of Poitiers - ISAE-ENSMA, France \\ *Corresponding author: michel.daaboul@balamand.edu.lb
}

\begin{abstract}
Airblast atomization is commonly used to atomize fuel in aircraft engines. An annular liquid sheet is atomized by the shear forces exerted by the co-flowing air stream. Nevertheless, this technique is less effective in some specific cases, e.g. when the external air flow velocity is relatively low. Electrohydrodynamic (EHD) atomization can constitute a solution in these cases. It consists of applying an electric field between two electrodes and electricallycharging the passing carburant. This phenomenon will create instabilities within the liquid, provoking therefore its atomization. The main objective is therefore to electrically atomize a liquid sheet without the application of an external air flow like in airblast atomizers.

This paper presents a novel actuator, based on dielectric barrier injection, used to induce instabilities within a plane liquid sheet of fuel similar to the annular sheet in aircraft engines. The behaviour of this atomizer was described in previous works. Several modes were observed, sometimes leading to a complete atomization, or just inducing instabilities and oscillating the liquid sheet. In the present study, only the cases where the liquid sheet is completely atomized are investigated. Images were recorded with the help of a high speed camera. Primary atomization is only studied, secondaryatomization being neglected. The properties of the spray obtained by EHD atomization are investigated thoroughly, namely the breakup length, the mesh size, the droplet diameter, the droplet count, etc.
\end{abstract}

\section{Keywords}

Atomization, Atomizers, Dielectric Barrier Injection, Electrohydrodynamics, Liquid Sheet.

\section{Introduction}

The phenomenon of atomization [1],[2] occurs through the interaction of a liquid in motion with respect to a surrounding gas. This occurrence results in the disintegration of the liquid in the form of droplets or fragments. Previous works [3] showed the importance of developing instabilities at the gas/liquid interface and thus leading to the liquid fragmentation.

Understanding the atomization of a liquid consists of understanding the processes that cause the conversion of a compact volume of liquid to a set of droplets [4]. The resulting spray is characterized by a distribution of diameters of the drops that compose it. There are many methods for disintegrating a liquid [5]. Atomizing a liquid sheet is a very efficient technique to obtain such sprays. The sheet thickness plays a major role during its atomization.

Whether it is from a jet [6],[7] or a sheet [8], a droplet is the ultimate step in the liquid fragmentation. The process involves several steps. When the liquid is initiallyin the form of a sheet, a sheet-ligament transition precedes the ligament-drops transition. The formation and the geometry of the ligaments depend on several parameters such as the thickness of the sheet or the speed of the surrounding flow.

At low speed, for example, aerodynamic forces exerted on the sheet produce longitudinal surface waves of great amplitude causing the sheet to beat like a flag. These instabilities lead to the formation of drops which size is of the order of magnitude of that of the sheet edge. The origin of these waves, which correspond to a phase displacement of the two interfaces, results from a Kelvin-Helmholtz type shear instability.

At higher speeds, the amplitude of the longitudinal waves becomes smaller and their frequency greater. The appearance of transverse ripples induces transverse fragmentation of the sheet in form of filaments, which leads to the formation of smaller drops.

The atomization of a liquid conventionally comprises two steps: the primary atomization [9] which corresponds to the formation of spherical or non-spherical liquid fragments, and the secondary atomization which refers to the additional fracture of the liquid fragments, resulting from the primary atomization, into finer droplets. The most cited model investigating secondaryatomization is that of Pilch and Erdman [10]. They present a model based on Ohnesorge number and Weber number values. In the current study, only primary atomization is investigated. 


\section{Material and Methods}

The current work presents experimental results obtained on a plane sheet of fuel. The experimental setup is shown in Figure 1.

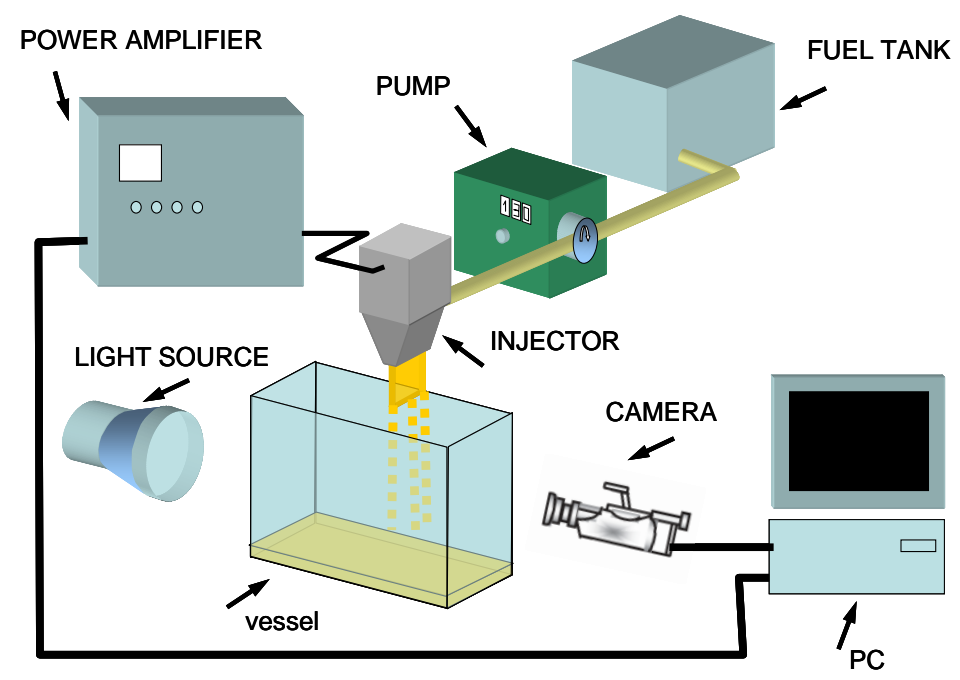

Figure 1. Experimental Setup.

The fuel (Diesel oil) is sucked in through a gear pump and then sent to the injector. The flow is controlled by the pump and adjusted bya manual table. The sheet velocity is therefore regulated by the fuel flow rate. The spray is collected in a vessel placed under the injector. It is also equipped with an anti-splash system (not shown in figure) which prevents the drops created by impact on the bottom of the container from disturbing the measurements. A high voltage square AC signal is provided by a power amplifier connected to the injecting electrode of the fuel injector. Electric signal frequency varies between $1 \mathrm{~Hz}$ and $2000 \mathrm{~Hz}$, and voltage goes up to $30 \mathrm{kV}$ in amplitude. The liquid sheet is filmed as it leaves the injector. A shadow ombroscopy measurement system is installed in order to be able to take instantaneous images of the sheet and study its behaviour with the application of the electric signal. A high speed camera takes up to 5000 images per second of the fuel sheet at a resolution of $640 \times 480$ pixels. The camera resolution could be increased to $1280 \times 960$ pixels but with lower image rate. The captured images are analysed and investigated on a pc.

The fuel injector shown in Figure 2 mainlyconsists of the dielectric material (1). Fuel (2) enters a surge chamber (3) to smooth out turbulence before entering a rectangular slit (4). The blades (5-6) are installed on the tip and allow obtaining a plane sheet (7), having a thickness of $300 \mu \mathrm{m}$ and a width of $62 \mathrm{~mm}$ into the paper. The velocity of the liquid sheet leaving the nozzle ranges between $0.6 \mathrm{~m} / \mathrm{s}$ (which is the minimum speed obtained with the pump) and $2 \mathrm{~m} / \mathrm{s}$. The counter electrode of the electrohydrodynamic actuator (8) is placed inside the injector. Electric charges are injected into the sheet at the level of the electrodes of the actuator.

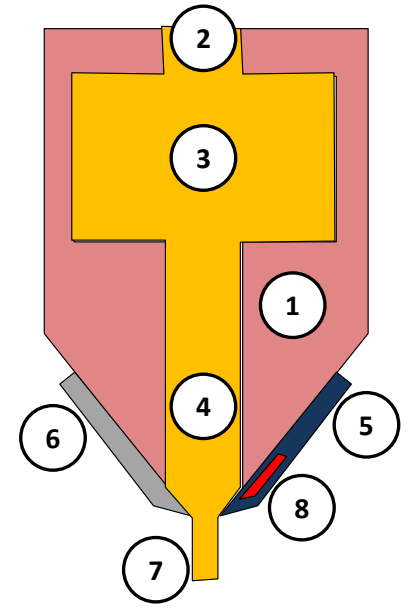

Figure 2. Schematic view of the fuel injector. 
Because such actuators are mainlyused in aircraft engine, kerosene would be the ideal fluid for this experiment. However, a fluid with close properties to kerosene ones is used in the experiments. It is commercial Diesel oil, a slightly conductive dielectric liquid. Its characteristics at a temperature of $20^{\circ} \mathrm{C}$ are presented in Table 1.

Table 1. Typical Characteristics of the Diesel Oil at $20^{\circ} \mathrm{C}$.

\begin{tabular}{|l|l|}
\hline Mass density & 850 \\
\hline Kinematic viscosity & $4.3 \cdot 10^{-0}$ \\
\hline Electrical conductivity & $1.15 \cdot 10^{-9}$ \\
\hline Relative permittivity & 2.2 \\
\hline
\end{tabular}

\section{Experimental Results}

When the fuel pump is running, a plane liquid sheet leaves the injector at a width of $62 \mathrm{~mm}$. This width does not remain constant because of surface tension effects. At the boundaries of the liquid sheet, two thick fragments grow as the sheet width decreases. It is shown on the left image of Figure 3. When an appropriate electric voltage is applied on the electrodes, instabilities are generated within the liquid and the sheet is atomized. It is visible on the right image of Figure 3. The development of the flow shows four regions until reaching a spray. First, primary oscillations of high frequency occur in the vicinity of the electrodes. The frequency of these oscillations is probably equal to the electric signal frequency. The second region is characterized by several local perforations of the sheet. Holes bounded by rims appear within the liquid. As these holes grow in size and in number, ligaments are produced and this third region is very close to a net or a mesh. In the lastregion, the ligaments break into droplets of different diameters. The distance from the injector lips until the start of ligaments breakup is defined as the breakup length. Detailed description and investigation on the processes leading to and occurring during atomization were presented in a previous work [11].
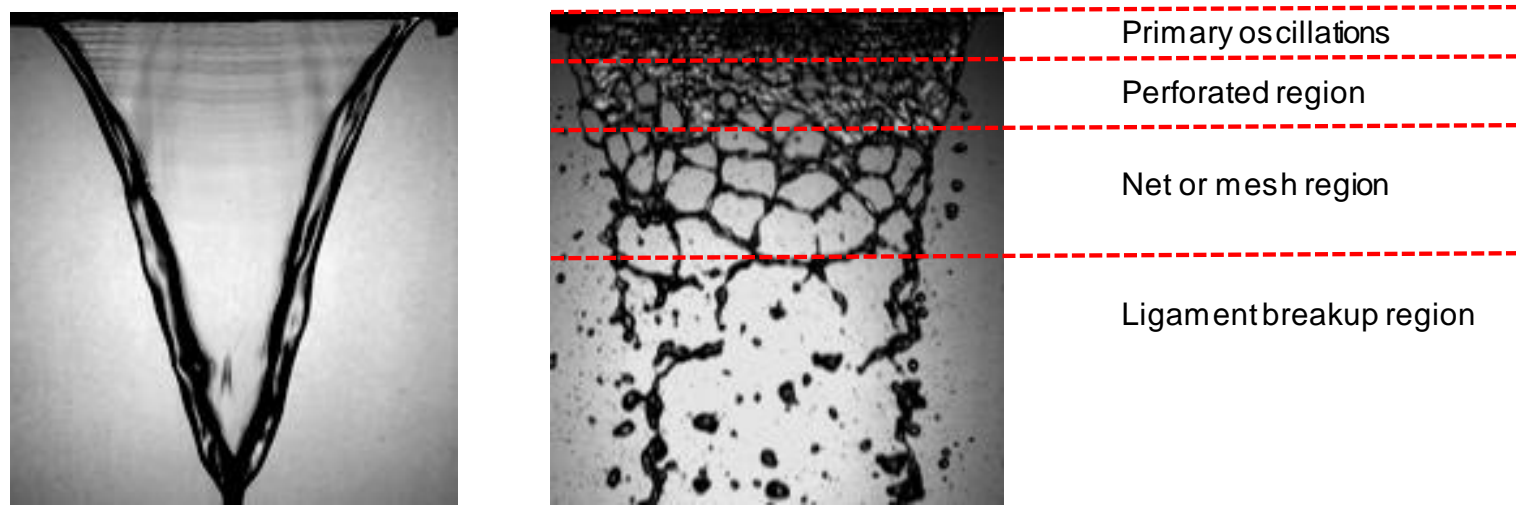

Figure 3. Shadow images of the plane sheet: left image $w$ ith no signal, right image $w$ hen atomized. (actual image size: $66 \mathrm{~mm}$ width, $73 \mathrm{~mm}$ height)

Figure 4 shows images of the liquid sheet for various electric signal frequencies and several liquid velocities. In the absence of electric signal, two edges are visible in black on each side of the liquid sheet. They are formed of a filament of liquid of large diameter. The edges of the sheet meet at few centimetres from the injector (seen in some images). Further downstream (outside the image frame) the sheet quickly turns into a cylindrical jet.

There are numerous physical parameters which can influence the spraying of the liquid sheet: liquid velocity, viscosity, density, air velocity, sheet thickness, etc. To all these mechanical parameters, add the set of electrical parameters: difference of potential, signal shape, signal frequency, polarity, electrode geometry, etc. It is nearly impossible to studyall the interactions resulting from the variations of all these parameters. To limit the number of parameters to be studied, the work presented here is conducted on a single sheet thickness ( $300 \mu \mathrm{m}$ at the exit of the injector) and just one electrode geometry is tested. All tests are carried out without external air flow, which means that atomization is a result of only electrohydrodynamic phenomena. Despite these limitations, the number of parameters and interactions to be studied remains very important. At this stage, it is preferred to conduct a qualitative study rather than a quantitative study [12]. Note that many other frequency values were tested but their corresponding images are not presented in this paper. 

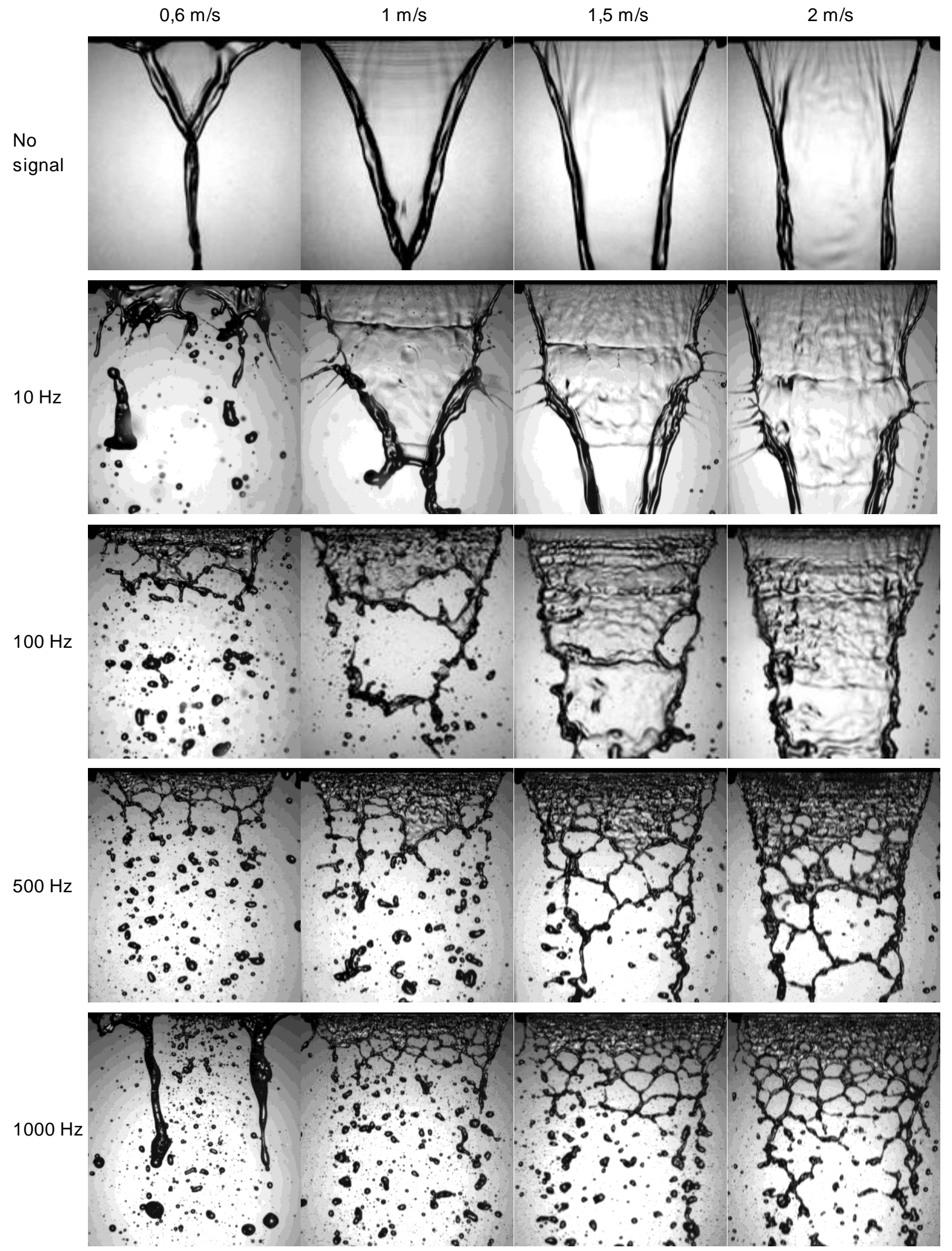

Figure 4. Experimental images of the liquid sheet behaviour $w$ hen exposed to various electric signal frequencies, and for various liquid velocities. Square AC signal, applied voltage $U= \pm 30 \mathrm{kV}$. (actual image size: $66 \mathrm{~mm} w$ idth, $73 \mathrm{~mm}$ height) 


\section{Interaction between Liquid Sheet Velocity and Electric Signal Frequency}

In previous works [11],[13], three modes of disturbance were observed: a flapping mode where the sheet oscillates like a flag, a disturbed mode where surface vibrations are visible, and a perforated mode where the sheet is pierced so that a mesh and/or ligaments are seen. The last mode only results in an atomization of the sheet.

The spraying mechanisms appear to be different depending on the frequency of the signal. A low frequency behaviour (frequency less than or equal to $100 \mathrm{~Hz}$ ) and a high frequency behaviour (above $300 \mathrm{~Hz}$ ) are distinguished. The transition between the two modes of operation is not immediate but ranges from $100 \mathrm{~Hz}$ to $300 \mathrm{~Hz}$, in which both behaviours can be observed.

At low frequency: The zone of primary oscillation is very extensive. In this zone a longitudinal wave with a frequency identical to that of the signal appears. This wave leads to the appearance on the sheet of thick transverse beads (two per period). The distance between the beads is halved when the frequency is doubled. Their thickness also decreases sharplywhen the frequency increases. The mesh area is practically non-existent at low sheet velocity. The breakup of the sheet occurs by piercing the membrane which connects the beads. They then become filaments. The collapse of these filaments produces a set of drops of large diameter.

At high frequency: The primary zone is reduced considerably, the oscillation takes a much more chaotic aspect and the beads are no longer visible. The mesh area becomes visible. The mesh size is then different according to the form of applied signal. Several shapes of AC electric signals were tested (square, triangle, sine, step) but only square signal results are presented in this paper. It is also observed that the mesh size decreases as the frequency increases.

\section{Study of the Breakup Length}

The breakup length is an important parameter when studying the behaviour of the liquid sheet. It can be easily measured on the previous images. Despite the high measurement uncertainty (especially at low frequency), very clear trends can be deducted. A set of 1000 images was captured for each case, and then the average value is presented in this work. In Figure 5, the behaviour of the breakup length as a function of the electric signal frequency is presented first (left chart). Then the behaviour is plotted versus the liquid sheet velocity (right chart). First, in the absence of electric signal, the sheet closes more or less rapidly. The lower the sheet velocity, the faster it closes.
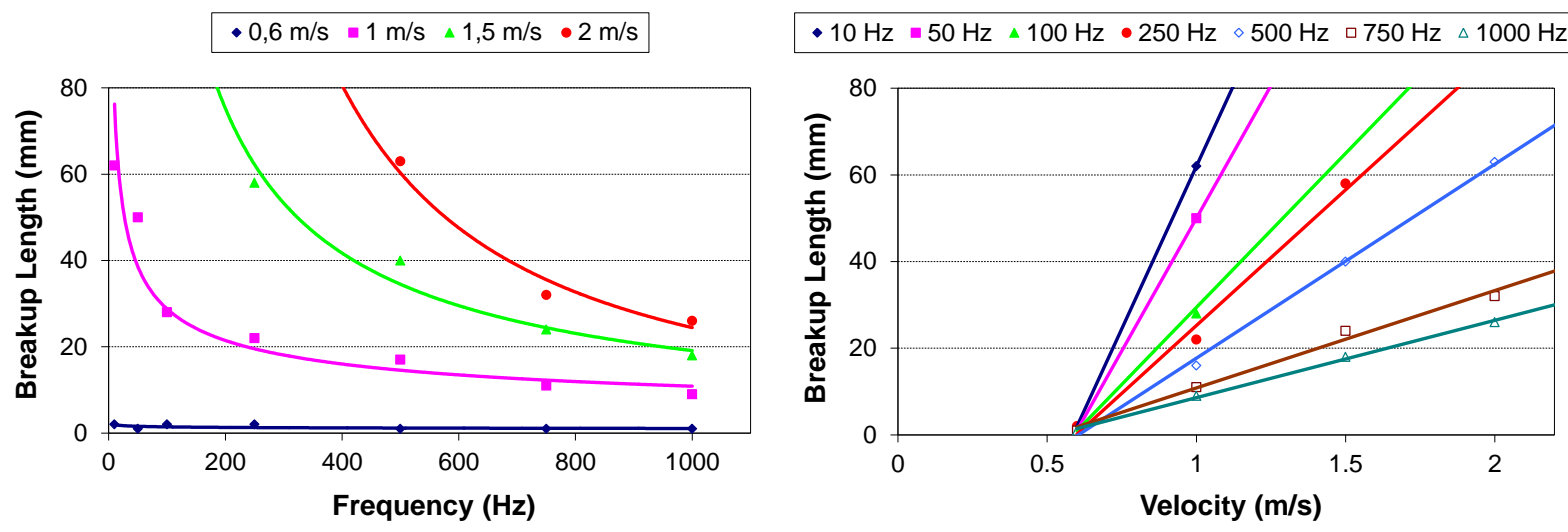

Figure 5. Breakup length variations versus electric signal frequency (left) and versus liquid sheet velocity (right).

A first observation is that the breakup length of the fuel sheet decreases as the electric signal frequency increases. This is due to the instabilities created within the sheet by EHD. At a frequency of $50 \mathrm{~Hz}$, the sheet is not always perforated. It is from the $100 \mathrm{~Hz}$ value that a breakup begins to be observed for the low sheet velocities. The breakup length decreases with increasing the frequency to a value of $7 \mathrm{~mm}$ at $1000 \mathrm{~Hz}$, at $1 \mathrm{~m} / \mathrm{s}$ sheet velocity for example. The decrease is asymptotic, and shorter breakup lengths could be obtained for frequencies over $1000 \mathrm{~Hz}$. The start of the perforation process depends strongly on the sheet velocity.

On the second chart, although curves are plotted with few points, the behaviour of the breakup length appears to be linear with the liquid velocity. Note that the breakup of the sheet occurs very close to the injector lips when the sheet velocity is lowest $(0.6 \mathrm{~m} / \mathrm{s})$. The dynamics of the mechanis $\mathrm{ms}$ that lead to the piercing of the liquid sheet appear to be independent of the sheet velocity. Similar behaviour was also observed for the mesh size. 


\section{Granulometric Study}

Figure 6 presents zoomed images of front views and side views of the liquid sheet when it is fully atomized at frequencies above $100 \mathrm{~Hz}$. The objective here is to conduct a granulometric studyon the droplet size and count. It is clearly visible on images that the frequency affects significantly the atomization, even for fully atomized sheets.

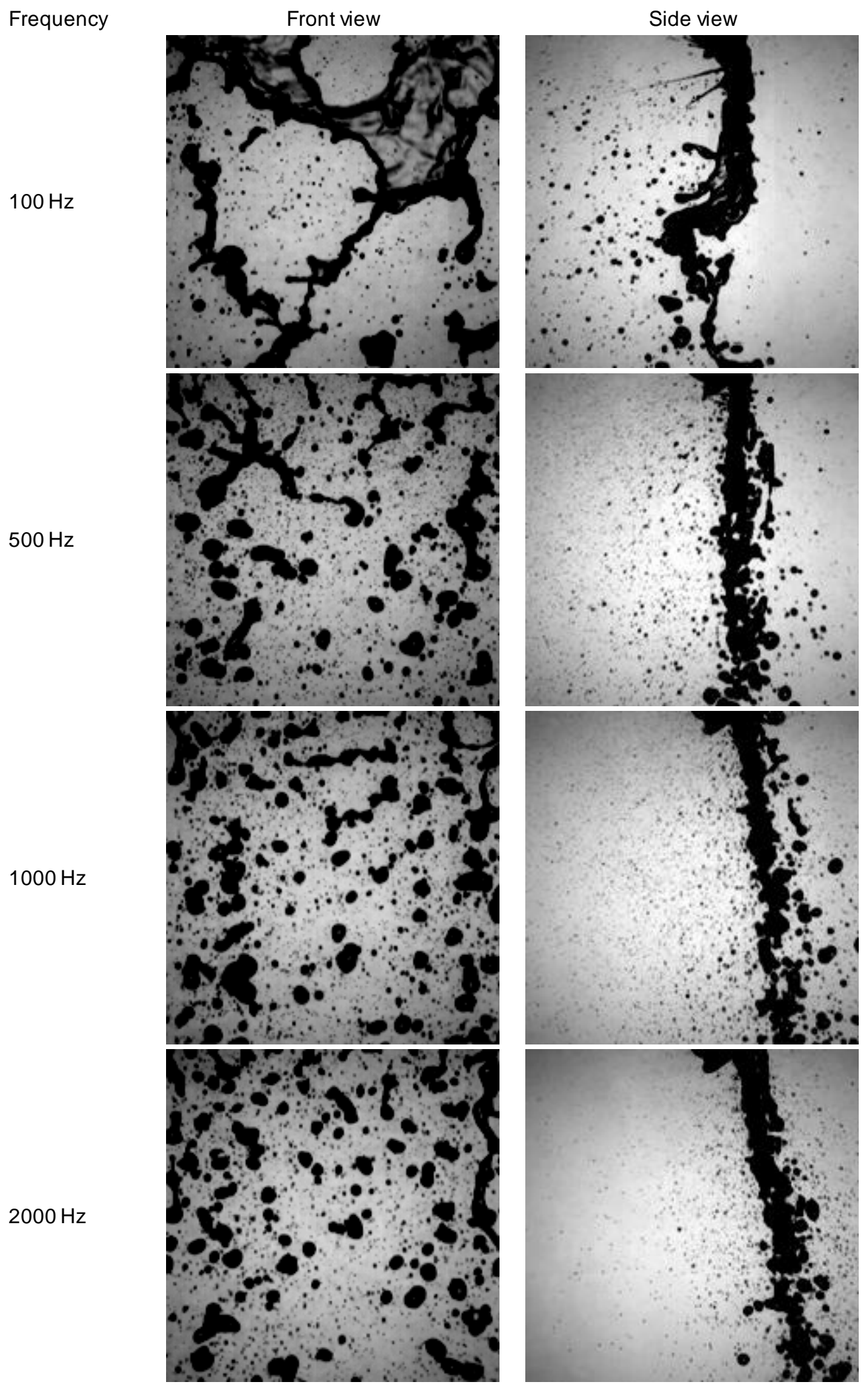

Figure 6. Zoomed images of the atomized sheet at various signal frequencies. (actual image size: $50 \mathrm{~mm}$ width, $50 \mathrm{~mm}$ height) 
If a higher zoom is applied on the images, the smallest drops will appear. A moderate frequency of $100 \mathrm{~Hz}$ leads to the formation of large structures as seen in Figure 6. Between these large structures, smaller drops are clearly visible. As the frequency increases, these drops become smaller and more numerous.

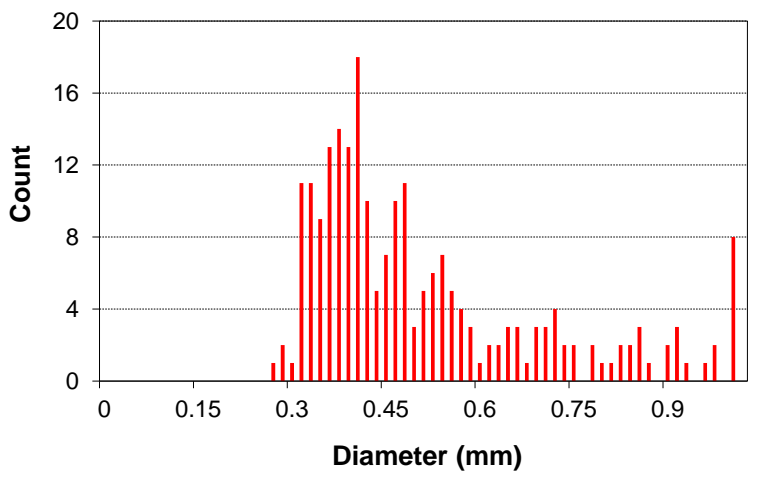

a. $100 \mathrm{~Hz}$

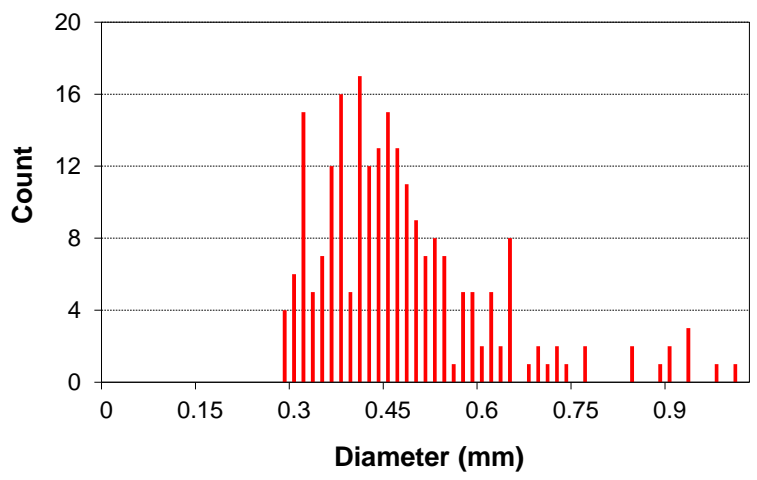

c. $1000 \mathrm{~Hz}$

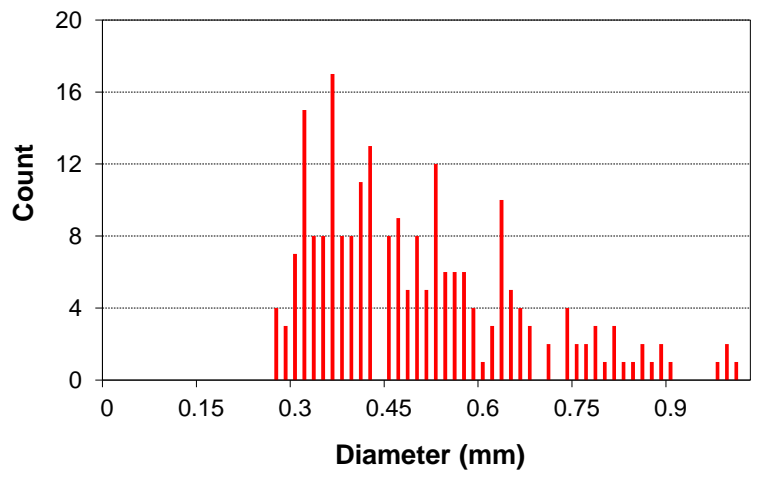

b. $500 \mathrm{~Hz}$

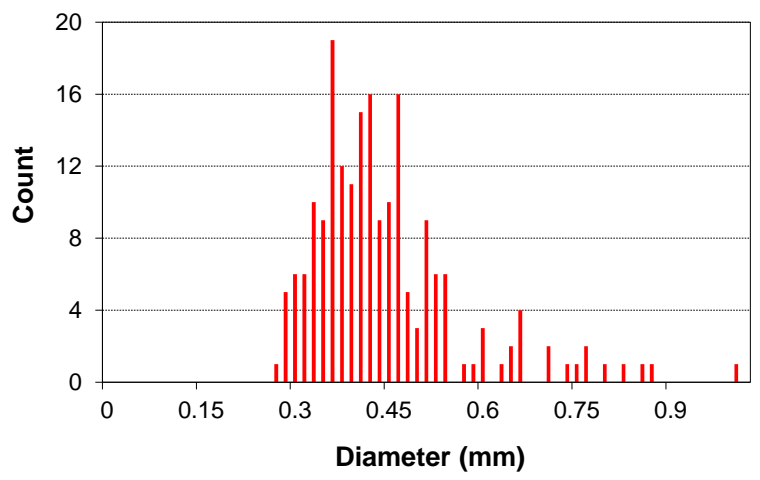

d. $2000 \mathrm{~Hz}$

Figure 7. Histograms of the count of droplet size for various frequencies.

Figure 7 presents the histograms of droplet count having various diameters, and for different frequencies of the electric signal. The software used for this treatment is Davis 7.0 software which is mainly used in Particle Image Velocimetry (PIV) measurements. Unfortunately, because of the dimensions of the images, the software did not allow us to detect drops whose diameter is less than $300 \mu \mathrm{m}$. Drops with a diameter greater than $1 \mathrm{~mm}$ are not rounded. In the case presented here, the liquid sheet is plane and large drops mostly result from the two rims bounding the sheet. On the contrary, in aircraft engines, fuel sheets are cylindrical and this problem does not occur. Therefore these large drops are not counted either.

The granulometric study is imperfect because manydrops are not detected by the software. Nevertheless, as the procedure executed on the images is exactly the same, the trends emerge. At $100 \mathrm{~Hz}$, the distribution of the drops is between 0.3 and $1 \mathrm{~mm}$. When the frequency increases, drops between 0.6 and $1 \mathrm{~mm}$ almost disappear. Therefore, the increase in frequencyseems to reduce the size of the drops. This study must be reconducted with another software appropriate for granulometry. In addition, a more thorough study on the Sauter mean diameter $D_{32}$ [14] should be carried out in order to obtain a more comprehensible behaviour of the atomization.

\section{Conclusions}

In this study, an EHD actuator was used to destabilize a sheet of Diesel fuel and lead to its atomization. The sheet was $62 \mathrm{~mm}$ wide and $300 \mu \mathrm{m}$ thick. Velocities ranging from $0.6 \mathrm{~m} / \mathrm{s}$ to $2 \mathrm{~m} / \mathrm{s}$ and electric signal frequencies ranging from $1 \mathrm{~Hz}$ to $2000 \mathrm{~Hz}$ were tested. A square $\mathrm{AC}$ electric signal with an amplitude of $\pm 30 \mathrm{kV}$ was applied between the electrodes to induce the liquid sheet atomization.

The possibilities offered by the EHD system have proved particularly rich. Two mechanisms of primary atomization seem to exist: a fast mechanism that pierces the sheet and creates a first set of small drops and then a slower mechanism, which corresponds to the disintegration of the mesh-ligaments and drops of millimetre size. 
It was observed that the frequency of the applied electric signal is an important parameter affecting the atomization process. High frequencies result in much better atomization. In addition, the liquid sheet velocity has an influence on the disintegration of the sheet. Higher liquid velocities require greater instabilities to completely atomize the liquid sheet.

The analysis of the images using an ombroscopy software allowed us to conduct a coarse granulometric study. The particle size study of EHD sprays, although carried out in a very fragmentary way, shows a clear tendency for the large drops to disappear when the frequency of the signal increases. A more appropriate granulometry software must be used in order to get the full distribution of droplet sizes.

The work carried out here aimed at demonstrating the efficiency of the process and delimiting the fields of operation. A more precise study must be carried out now to quantify more accurately the influence of each of the parameters governing the atomization process.

Finally, after comparison of the obtained results to those of air-blast atomization, it would be interesting to investigate the combined effect of electrohydrodynamic and air-blast atomizations in future experiments.

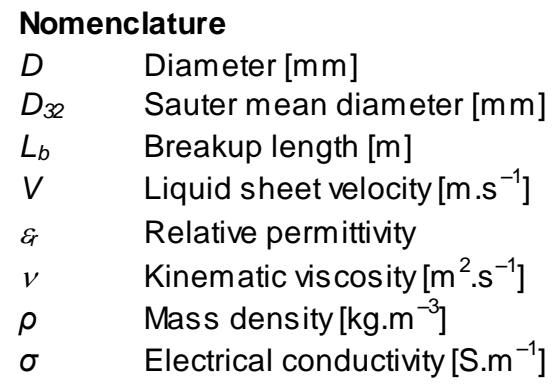

\section{References}

[1] Mansour, A., Chigier, N., Disintegration of liquid sheets, 1990, Physics of Fluids A, 3, pp. 2971-2980.

[2] Mansour, A., Chigier, N., Dynamic behavior of liquid sheets, 1991, Physics of Fluids A, 2, pp. 706-719.

[3] Berthoumieu, P., Carentz, H., Experimental study of a thin planar liquid sheet disintegration, July 16-20, 2000, $8^{\text {th }}$ International Conference on Liquid Atomization and Spray Systems, ICLASS 2000, Pasadena, CA, USA.

[4] Berthoumieu, P., Lavergne, G., Video techniques applied to the characterization of liquid sheet breakup, 2001, Journal of Visualization, 4 (3), pp. 267-275.

[5] Larricq-Fourcade, C., Lavergne, G., Berthoumieu, P., Thivet, F., Improvement of airblast injectors with electro-aerodynamic actuators, October $3-7,2005,2^{\text {nd }}$ International Symposium on Nonequilibrium Processes, Plasma, Combustion and Atmospheric Phenomena, NEPCAP 2005, Sochi, Russia.

[6] Shrimpton, J.S., Electrohydrodynamics of charge injection atomization: Regimes and fundamental limits, 2003, Atomization and Sprays, 13 (2\&3).

[7] Rigit, A.R.H., Shrimpton, J., Electrical performance of charge injection electrostatic atomizers, 2006, Atomization and Sprays, 16 (4), pp. 401-420.

[8] Bremond, N., Clanet, C., Villermaux, E., Atomization of undulating liquid sheets, 2007, Journal of Fluid Mechanics, 585, pp. 421-456.

[9] Dumouchel, C., On the experimental investigation on primary atomization of liquid streams, 2008, Experiments in Fluids, 45, pp. 371-422.

[10] Pilch, M., Erdman, C.A., Use of the breakup time data and velocity history data to predict the maximum size of stable fragments for acceleration induced breakup of a liquid drop, 1987, International Journal of Multiphase Flow, 13, pp. 741-757.

[11] Louste, C., Daaboul, M., Traore, Ph., Romat, H., Experimental Study of electrical primary break-up of a thin sheet of dielectric liquid controlled by an electrohydrodynamic actuator, September 6-9 2010, 23 ${ }^{\text {rd }}$ European Conference on Liquid Atomization and Spray Systems, ILASS-Europe 2010, Brno, Czech Republic.

[12] Lastow, O., Balachandran, W., Numerical simulation of electrohydrodynamic (EHD) atomization, 2006, Journal of Electrostatics, 64 (12), pp. 850-859.

[13] Louste, C., Daaboul, M., Traoré, P., Romat, H., Influence of signal properties on electrohydrodynamic primary break-up of thin sheets of dielectric liquid, September 2-6, 2012, $12^{\text {th }}$ International Conference on Liquid Atomization and Spray Systems, ICLASS 2012, Heidelberg, Germany.

[14] Sauter, J., Die Grössenbestimmung der im Gemischnebel von Verbrennungskraftmaschinen vohrhandenen Brennstoffteilchen, 1926, Mitteilung aus dem Laboratorium für Technische Physik der Technischen Hochschule München, VDI-Verlag. 